\title{
Power evaluation of advanced energy-harvester using graphical analysis
}

\author{
Kanjuro MAKIHARA*, Akihiro TAKEZAWA**, Daisuke SHIGETA* and Yuta YAMAMOTO* \\ *Department of Aerospace Engineering, Tohoku University \\ 6-6-01 Aramaki-Aza-Aoba, Aoba-Ward, Sendai, Miyagi 980-8579, Japan \\ **Division of Mechanical Systems and Applied Mechanics, Institute of Engineering, Hiroshima University \\ 1-4-1 Kagamiyama, Higashi-Hiroshima, Hiroshima 739-8527, Japan \\ E-mail: makihara@ssl.mech.tohoku.ac.jp
}

\section{Received 17 October 2014}

\begin{abstract}
In this paper, we present the benefits of bond-graph analysis for mechanical-electrical systems, which are energy-harvesters based on structural vibrations and electric loads. The bond-graph is an energy-based approach to describing physical-dynamic systems. It shows power flow graphically, which helps us understand the behavior of complicated systems in simple terms. Energy-harvesting involves conversion of power in mechanical form to the electrical one and the bond-graph is a good tool to analyze this flow. The bond-graph method can be used to calculate the dynamics of the combining mechanical and electrical systems simultaneously. The biggest advantage of the bond-graph technique is that it can be used with the systems that are subject to component alternations, such as inserting, removing and swapping. The bond-graph method involves solving simultaneous algebraic equations, instead of differential equations. On the other hand, in common simulation methods, such as solving differential equations, it is difficult to change the number of components because the differential equations will have to be reconstructed. Because the bond-graph has not been used for harvesting analysis, bond-graph models for harvesting need to be created in advance of numerical analysis. In this paper, we first proposed a piezoelectric model that matches the bond-graph method. We also propose a diode-bridge model and a harvesting controller model that are suitable for bond-graph analysis. We then analyze a self-powered energy harvester that has multi-bifurcated and looped flow in the mechanical-electrical coupled dynamics.
\end{abstract}

Key words: Bond-graph, Energy-harvesting, Energy-harvester, Piezoelectric transducer, Mechanical-electrical coupled, Power analysis

\section{Introduction}

Over the years, there has been a growing interest in energy harvesting technology. This technology extracts electrical energy from different forms of energy in the environment such as solar energy, wind energy, heat energy, and vibration energy. Energy harvesting is also called environmental power generation and has the benefit of not burdening the environment. There are many structures that display mechanical vibrations. Therefore, vibration-based energy harvesting is of interest for many researchers.

Many studies on vibration-based energy harvesting use piezoelectric-material based techniques to harvest the vibration energy (Adachi and Sakamoto, 2012, Dicken, et al., 2012, Masuda, et al., 2013, Caliò, et al., 2014). Especially, the synchronized switch harvesting on inductor (SSHI) technique is of interest, because SSHI harvests vibration energy with a high efficiency by using an inductor and an on/off switch (Badel, et al., 2005). The SSHI technique was improved by reducing the power-consumption of device (Makihara, et al., 2006). However, SSHI needs a controller for switching-control. The control device is power intensive and requires an external power supply. Therefore, it is not very suitable for energy-harvesting applications. To work around this problem, an analog functional unit, which does not need a power supply and does not have a large consumption, was used (Niederberger and Morari, 2006). But this 
analog technique also has demerits. It is difficult to change its parameters after packaging and it is impossible to make complex calculation. Therefore, an innovative harvester employing a digital functional unit including a digital processor was invented (Makihara, et al., 2012a, Makihara, et al., 2012b, Yamamoto and Makihara, 2014). The digital functional unit made complex calculations possible making it is suitable for harvesting energy from complex vibrations. Because of the low power-consumption of the digital functional unit, it can be driven by harvested energy, making it a self-powered system that does not need an external power supply. However, the circuits in the harvester become very complicated, which makes it difficult to analyze its performance with conventional ways of computational simulation.

The bond-graph is a power-based approach for describing physical-dynamic systems (Karnopp, et al., 1990). It shows power flow graphically, which helps us analyze the behavior of complicated systems in simple terms. The harvesters, including piezoelectric transducers, display a bidirectional power flow between the mechanical and electrical fields. Thus, the bond-graph is suitable for dynamic analysis of the harvester. To analyze the harvesters, we need a model of the piezoelectric transducer. In the past, many bond-graph models of the piezoelectric transducer have been proposed. In these models, the piezoelectric transducer was discussed in purely circuit terms and it did not capture the mechanical dynamics well. A proposal by Mason et al. (1948) used a transducer to separate the mechanical field from the electrical field; however, energy equations of this model were not accurate. Busch-Vishniac and Paynter (1991) proposed a variable-capacitor model. This model's energy equations were accurate, but the model was too complicated to simulate systems with piezoelectric transducers. Wang et al. (2009) proposed a model for the piezoelectric material that was easy to use in simulations. Because the model displayed unnecessary energy losses, it did not gain much traction. A simple model was proposed by Rodriguez-Fortun et al. (2010), but they only proposed the idea and did not demonstrate the usability of their model.

\section{Problem statement and research objective}

Figure 1 shows a conceptual illustration of our self-powered energy harvester (Makihara, et al., 2012a, Makihara, et al., 2012b, Yamamoto and Makihara, 2014). When the structures vibrate, a piezoelectric transducer generates an electric potential difference. The potential difference is rectified through a diode-bridge, and it will be stored in a storage capacitor. The stored voltage gradually increases owing to the structural vibration. A digital processor can be driven by the energy harvested from structural vibration. The digital processor receives the information about the structural displacement from a sensor that is a small piezoelectric element and attached to the structure. The sensor generates a voltage according to the displacement. The digital processor calculates the time when it has to regulate the switches in the harvester. A digital processor intermittently outputs a control signal to the switches. Then, the smart energy harvester achieves highly efficient energy harvesting completely without any power supply and control management from the outside. Consequently, this energy harvesting system can be considered a self-powered device.

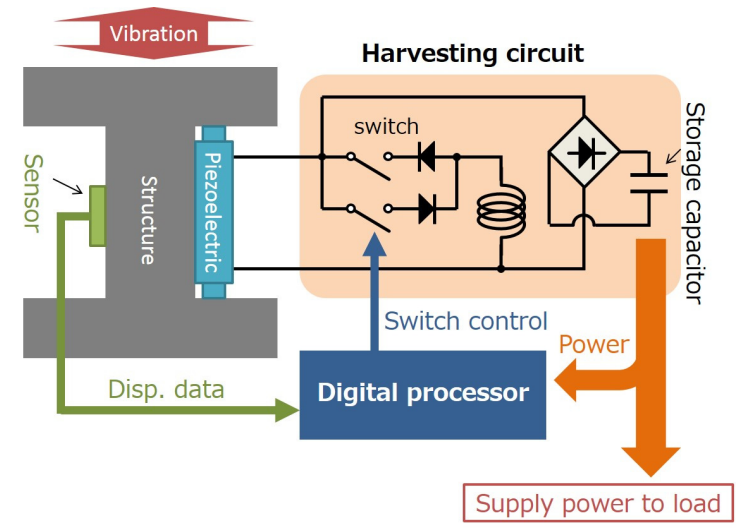

Fig. 1: Concept of the self-powered harvester.

However, the circuits in the harvester become very complicated, which makes it difficult to analyze its performance using the conventional method of computational simulation. In order to develop high-efficiency harvesters, a performance analysis of the harvesters is important. The self-powered harvester has a multi-bifurcated and looped power flow that requires a flexible and advanced simulation method for the power analysis. In order to evaluate the 
proposed self-powered energy harvester, we simulate it using the bond-graph technique. We develop a piezoelectric transducer model that matches the bond-graph method. A bond-graph model of the self-powered energy harvester is created to evaluate the performance of the harvester from a power viewpoint. To the best of our knowledge, there have not been power analyses of energy harvesters using the bond-graph calculation. Since the bond-graph has not been used extensively for harvesting analysis, bond-graph models for harvesting need to be created in advance of bond-graph analysis. From the research presented in this paper, the usability and versatility of the bond-graph is demonstrated for its more extensive usage.

\section{Bond-graph method}

A physical system is described in terms of effort and flow. In the mechanical field, the force is the effort and velocity is the flow. In the electrical field, voltage is the effort and current is the flow. Effort and flow are called power variables. Power is equal to the product of flow and effort. The energy is calculated by integrating power with respect to time. There are two energy variables. The first one is called the momentum and is calculated by integrating effort with respect to time. The second one is called the displacement and is calculated by integrating flow with respect to time. Table 1 shows the different variables in the physical field.

Table 1 Power and energy variables in mechanical and electrical fields.

\begin{tabular}{cccc}
\hline \hline & Mechanical rotation & Mechanical translation & Electricity \\
\hline Effort & Force & Torque & Voltage \\
$(e)$ & $F[\mathrm{~N}]$ & $\tau[\mathrm{N} \cdot \mathrm{m}]$ & $V[\mathrm{~V}]$ \\
\hline Flow & Velocity & Angular velocity & Current \\
$(f)$ & $\dot{x}[\mathrm{~m} / \mathrm{s}]$ & $\omega[\mathrm{rad} / \mathrm{s}]$ & $i[\mathrm{~A}]$ \\
\hline Momentum & Momentum & Angular momentum & Magnetic flux \\
$(p)$ & $P[\mathrm{~N} \cdot \mathrm{s}]$ & $p[\mathrm{~N} \cdot \mathrm{m} \cdot \mathrm{s}]$ & $\varphi[\mathrm{Wb}]$ \\
\hline Displacement & Displacement & Angle & Charge \\
$(q)$ & $x[\mathrm{~m}]$ & $\theta[\mathrm{rad}]$ & $Q[\mathrm{C}]$ \\
\hline
\end{tabular}

There are three basic components of the bond-graph: $C, I$ and $R$. The $C$ and $I$ elements are used to model a phenomenon that relates effort to displacement and flow to momentum. The $C$ element acts as a mechanical spring or an electrical capacitor (energy storage described by a generalized displacement variable). The $I$ element acts as a mechanical mass or an electrical inductor (energy storage described by a generalized momentum variable). The $R$ element is used to model a phenomenon that ties effort and flow. It is a power dissipative element and acts as a mechanical damper or an electric resistor. Two transformation elements also exist, the ideal transformer $T F$ and the gyrator $G Y$. The $T F$ element relates the primary effort to the secondary effort and the primary flow to the secondary flow. The $G Y$ element relates the primary effort to the secondary flow and the primary flow to the secondary effort. These elements are tied by a 0-bond or 1-bond. The 0-bond is the constant effort point, such as 2 elements associated with the same force or voltage. The 1-bond is the constant flow point, such as 2 elements associated with the same velocity or current. Table 2 lists the components of a bond-graph.

Table 2 Basic components of bond-graph.

\begin{tabular}{ccc}
\hline \hline Component & Exposition & Relation \\
\hline$I$ & Mass or inductor & $f=f_{\mathrm{I}}(p)$ \\
\hline$C$ & Spring or capacitor & $q=f_{\mathrm{C}}(e)$ \\
\hline$R$ & Damper or resistor & $e=f_{\mathrm{R}}(f)$ \\
\hline$T F$ & Transformer & $e_{1}=\alpha e_{2}, f_{2}=\alpha f_{1}$ and $\alpha=$ const. \\
\hline$G Y$ & Gyrator & $e_{1}=\beta f_{2}, e_{2}=\beta f_{1}$ and $\beta=$ const. \\
\hline 0 & Constant effort point & $e=$ const.and $\sum f=0$ \\
\hline 1 & Constant flow point & $f=$ const.and $\sum e=0$ \\
\hline
\end{tabular}


The biggest advantage of the bond-graph is that it can be applied to systems that change its components. The bond-graph solves characteristic and algebraic equations, instead of differential equations. Simulations that solve differential equations are not as effective in simulating these systems because the differential equations have to be reconstructed when the components change (i.e., inserting, removing or swapping components). For more details on bond-graphs, see Karnopp et al. (1990) and Thoma (1990).

\section{Model creation of harvesting components for bond-graph analysis}

As previously mentioned, some bond-graph models of the harvester's components need to be created. We consider three models: the piezoelectric-transducer model, the diode-bridge model, and the harvesting controller model. These models are used in the advanced energy-harvester. Since the bond-graph model of the advanced energy-harvester is very complex to build as a whole, we create the bond-graph models for individual elements. By assembling the individual models, we build the bond-graph model of the advanced energy-harvester.

In the first section, we evaluate the validity of the piezoelectric transducer model. First, we describe the piezoelectric transducer model, the constitutive equation, and the bond-graph representation. Second, we perform numerical simulation of a model that includes the piezoelectric transducer. We judge the validity of the simulation results from the energy conservation law. In the second section, we discuss the diode model and the diode-bridge model. The diode model is assumed to be an ideal model. In the third section, we describe the controller model.

\subsection{Verification of piezoelectric transducer model}

\subsubsection{Piezoelectric transducer model and constitutive equations}

So far, many piezoelectric transducer models have been proposed for various purposes, but most of them do not match the bond-graph method. Figure 2(a) shows the dynamic model of the piezoelectric transducer that we created, which matches the bond-graph. It consists of a mechanical spring $k_{\mathrm{t}}$, a mechanical-electrical transformer, and an electrical capacitor $C_{t}$. In the following description, $x$ is the absolute displacement of the node, $x_{1}$ is the elongation of the mechanical-electrical transformer, $x_{2}$ is the elongation of the mechanical spring, $F$ is the tensile force exerted on the piezoelectric transducer, $V$ is the voltage of the piezoelectric transducer, $V_{1}$ is the voltage of the capacitor, $V_{2}$ is the voltage of the mechanical-electrical transformer, $Q$ is the electric charge of a capacitor, and $i$ is the current through the piezoelectric transducer. The constitutive equations for the piezoelectric transducer can be expressed as

$$
F=k_{\mathrm{t}} x-b_{\mathrm{t}} Q, \quad V=-b_{\mathrm{t}} x+\frac{Q}{C_{\mathrm{t}}^{\prime}},
$$

where

$$
b_{\mathrm{t}} \equiv k_{\mathrm{t}} \alpha, \quad C_{\mathrm{t}}^{\prime} \equiv \frac{C_{\mathrm{t}} k_{\mathrm{t}}}{k_{\mathrm{t}}+b_{\mathrm{t}}^{2} C_{\mathrm{t}}},
$$

$b_{\mathrm{t}}$ is the piezoelectric coefficient, $\alpha$ is the mechanical-electrical transformation factor and $C_{\mathrm{t}}^{\prime}$ is the constant-strain of the piezoelectric transducer. A bond-graph representation of the piezoelectric transducer is shown in Fig. 2(b). The figure has a few bond-graph symbols: two $C$ elements represent the mechanical spring and the electrical capacitor, TF element represents the energy converter, 0 indicates the constant-effort variable point, namely constant-force point, and 1 indicates the constant-flow variable point, namely constant-current point.

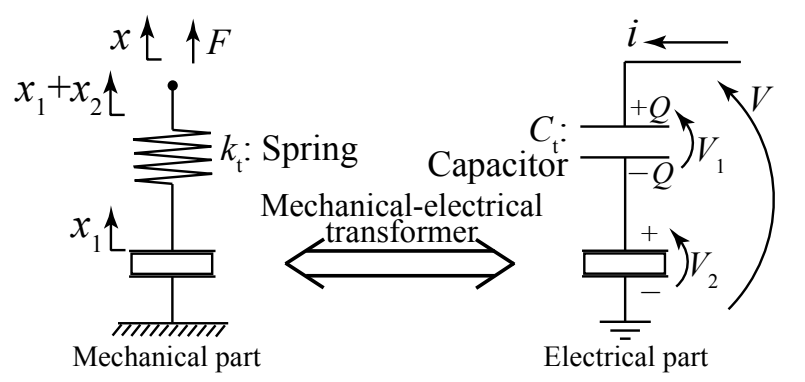

(a) Dynamic model

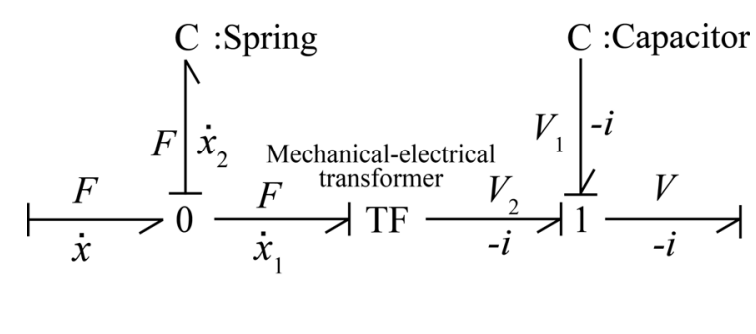

(b) Bond-graph representation

Fig. 2 Piezoelectric transducer model that matches the bond-graph method. 


\subsubsection{Numerical simulation of the system that has a piezoelectric transducer}

For testing the validity of the model in Fig. 2, we simulated the mechanical-electrical system shown in Fig. 3(a). In the analysis model example, we assumed a passive vibration control system including the piezoelectric transducer. Figure 3(b) shows a bond-graph representation of the model in Fig. 3(a). The system parameters used in the simulations are given in Table 3 .

Table 3 Parameters of Fig. 3(a) used in simulation.

\begin{tabular}{c|ccc}
\hline \hline Symbol & Quantity & Value & Unit \\
\hline$M$ & Mass & $1.50 \times 10^{1}$ & $\mathrm{~kg}$ \\
$k_{\mathrm{t}}$ & Constant-charge stiffness & $2.28 \times 10^{5}$ & $\mathrm{~N} / \mathrm{m}$ \\
$b_{\mathrm{t}}$ & Piezoelectric coefficient & $1.55 \times 10^{5}$ & $\mathrm{~V} / \mathrm{m}$ \\
$C_{\mathrm{t}}^{\prime}$ & Constant-strain capacitance & $6.90 \times 10^{-7}$ & $\mathrm{~F}$ \\
$L$ & Inductance & $9.53 \times 10^{1}$ & $\mathrm{H}$ \\
$R$ & Resistance & $3.16 \times 10^{4}$ & $\Omega$ \\
$\dot{x}$ & Initial velocity of mass & $1.10 \times 10^{-3}$ & $\mathrm{~m} / \mathrm{s}$ \\
\hline \hline
\end{tabular}

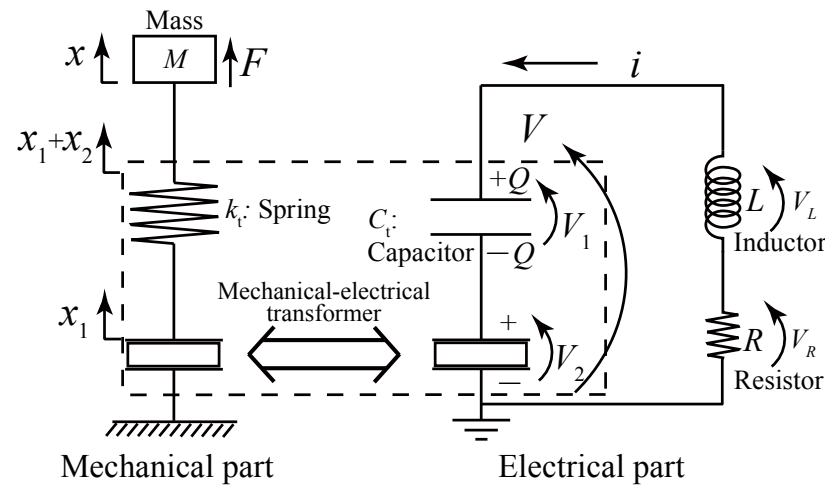

(a) Dynamic model

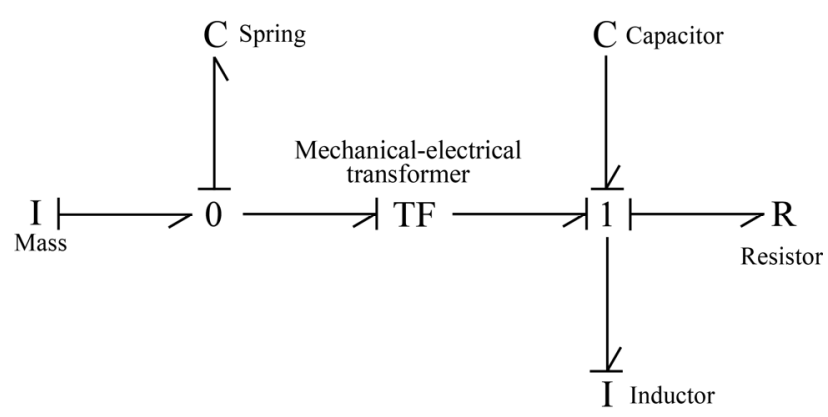

(b) Bond-graph representation

Fig. 3 Simulation model to verify the piezoelectric transducer model.

The inductance is chosen to match the electrical frequency with the mechanical frequency so that the system functions like a dynamic vibration absorber. The resistance is chosen to reduce the mechanical energy efficiently. The mass is in free vibration, i.e., it has an initial velocity and is not acted upon by an external force. To assess the validity of the simulation results, we apply the energy conservation law to the system using the constitutive equations of the piezoelectric transducer. When an external force $f_{\text {ext }}$ is applied to the mass, with Eq. (1), the motion equation can be expressed as

$$
M \ddot{x}+\left(k_{\mathrm{t}}-b_{\mathrm{t}}^{2} C_{\mathrm{t}}^{\prime}\right) x-b_{\mathrm{t}} C_{\mathrm{t}}^{\prime} V=f_{\mathrm{ext}} .
$$

When the external voltage $V_{\text {ext }}$ is input to the piezoelectric transducer, the circuit equation can be expressed as

$$
L \ddot{Q}+R \dot{Q}+V=V_{\text {ext }} \text {. }
$$

The work done by the external force and the external voltage between 0 and $t^{\prime}, W_{f_{\text {ext }}}$ and $W_{V_{\text {ext }}}$, respectively, can be defined as

$$
W_{f_{\text {ext }}} \equiv \int_{0}^{t^{\prime}} f_{\text {ext }} \cdot \dot{x} \mathrm{~d} t, \quad W_{V_{\text {ext }}} \equiv \int_{0}^{t^{\prime}} \dot{Q} \cdot V_{\text {ext }} \mathrm{d} t .
$$

The total work done $W$ by the system can be expressed by using Eqs. (3) - (5) as

$$
W \equiv W_{f_{\text {ext }}}+W_{V_{\text {ext }}}=\left[\frac{1}{2} M \dot{x}^{2}\right]_{0}^{t^{\prime}}+\left[\frac{1}{2}\left(k_{\mathrm{t}}-b_{\mathrm{t}}^{2} C_{\mathrm{t}}^{\prime}\right) x^{2}\right]_{0}^{t^{\prime}}+\left[\frac{1}{2} L \dot{Q}^{2}\right]_{0}^{t^{\prime}}+\left[\frac{1}{2} C_{\mathrm{t}}^{\prime} V^{2}\right]_{0}^{t^{\prime}}+\int_{0}^{t} R \dot{Q}^{2} \mathrm{~d} t .
$$

If we assume no external input, Eq. (6) becomes 


$$
\left[\frac{1}{2} M \dot{x}^{2}\right]_{0}^{t^{\prime}}+\left[\frac{1}{2}\left(k_{\mathrm{t}}-b_{\mathrm{t}}^{2} C_{\mathrm{t}}^{\prime}\right) x^{2}\right]_{0}^{t^{\prime}}+\left[\frac{1}{2} L \dot{Q}^{2}\right]_{0}^{t^{\prime}}+\left[\frac{1}{2} C_{\mathrm{t}}^{\prime} V^{2}\right]_{0}^{t^{\prime}}+\int_{0}^{t} R \dot{Q}^{2} \mathrm{~d} t=0
$$

Because the first four terms in Eq. (7) do not depend on time, these terms are regarded as energy. The last term depends on time and represents energy dissipation. Specifically, the first and the second terms are mechanical energy terms and the third and the fourth terms are electrical energy terms. The fifth term that depends on time is the dissipative energy. The energy terms are listed in Eqs. (8)-(10).

$$
\begin{gathered}
\text { Mechanical energy } \equiv \frac{1}{2} M \dot{x}^{2}+\frac{1}{2}\left(k_{\mathrm{t}}-b_{\mathrm{t}}^{2} C_{\mathrm{t}}^{\prime}\right) x^{2}, \\
\text { Electrical energy } \equiv \frac{1}{2} L \dot{Q}^{2}+\frac{1}{2} C_{\mathrm{t}}^{\prime} V^{2}, \\
\text { Dissipative energy } \equiv \int_{0}^{t} R \dot{Q}^{2} \mathrm{~d} t .
\end{gathered}
$$

When the system does not have external input, the sum of the energy terms in Eqs. (8) - (10) is constant.

Figure 4 shows the change in mechanical energy, electrical energy, dissipative energy and total energy with time. Mechanical energy decreases when it is converted to electrical energy by the piezoelectric transducer. The converted electrical energy is dissipated by the resistor, and it finally converges to zero. The decrease in mechanical energy indicates that the vibration amplitude of the mass is decreasing. In the simulation model, the system only has an initial velocity and no external input. Therefore, the initial total energy equals the kinetic energy of the mass. Thus, the total energy including the dissipative energy must always be constant. Since Fig. 4 indicates that the total energy in the model is a constant, it shows that the piezoelectric transducer model shown in Fig. 2 is valid. In a later section, we use the piezoelectric transducer model shown in Fig. 2.

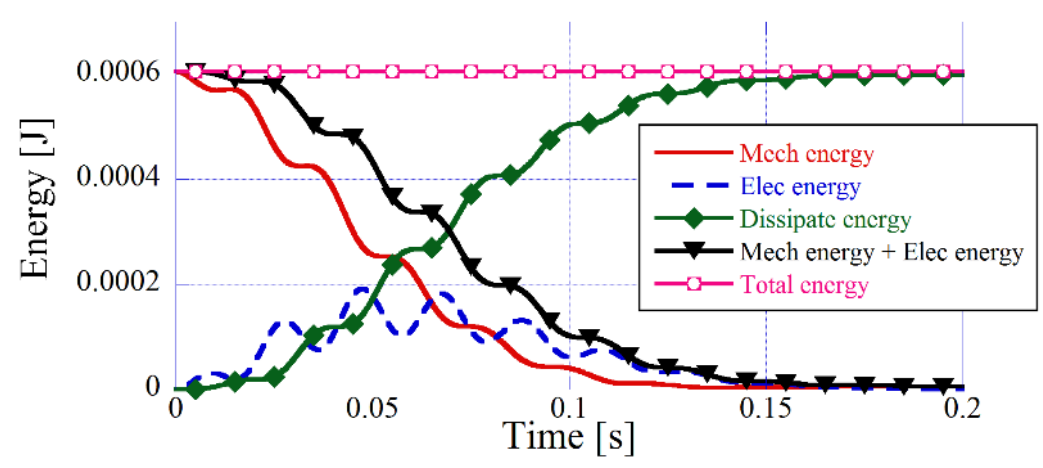

Fig. 4 History of various energies with passive method.

\subsection{Bond-graph model of diode-bridge \\ 4.2.1 Diode model}

In this section, we discuss the diode model including its forward voltage drop, inner resistor and rectifying effect. The diode model is divided into two states: one state allows the electric current to pass in one direction and the other state blocks electric current in the opposite direction. These states can be changed by an on/off switch. As shown in Fig. 5(a), it consists of a counter voltage that stands for the forward voltage drop $V_{\text {for }}$, an electrical resistor that indicates the internal resistance $R_{\mathrm{D}}$, and an on/off switch. With Heaviside function, the state of the on/off switch can be expressed as the follows:

$$
\mathrm{H}\left(V_{\mathrm{D}}-V_{\text {for }}\right) \equiv\left\{\begin{array}{l}
1\left(\text { when } V_{\mathrm{D}}-V_{\text {for }} \geq 0\right) \\
0\left(\text { when } V_{\mathrm{D}}-V_{\text {for }}<0\right)
\end{array}\right.
$$

where $V_{\mathrm{D}}$ is voltage across the diode and $V_{\text {for }}$ is the forward voltage drop. If the electric current without the on/off switch is $i_{\mathrm{o}}$, the electric current with the on/off switch can be expressed as

$$
i=i_{\mathrm{o}} \cdot \mathrm{H}\left(V_{\mathrm{D}}-V_{\text {for }}\right) \text {. }
$$


The forward voltage of diode $V_{\text {for }}$ is $0.6 \mathrm{~V}$ and $R_{\mathrm{D}}=1.0 \times 10^{2} \Omega$. Figure $5(\mathrm{~b})$ shows the bond-graph presentation of Fig. 5(a). The on/off action of the on/off switch is described by a variable resistor $R$ in Fig. 5(b). When the on/off switch turns on in Fig. 5(a), the resistance value $R$ in Fig. 5(b) becomes $R_{\mathrm{D}}$. When the on/off switch turns off, the resistance value $R$ in Fig. 5(b) becomes large enough to prevent electric current from flowing. The SE symbol, which is one of the bond-graph elements, represents the source-effort elements such as a voltage source. Actual diodes do not have any active elements, i.e., voltage sources. However, there have been some suggestions that passive diodes can be modeled with active elements (Jaeger and Blalock, 2010). The binary system sometimes causes chattering phenomenon within a short period after the resistance value changes. When we set the simulation time-step short enough, the chattering is not a serious problem for all practical purposes.

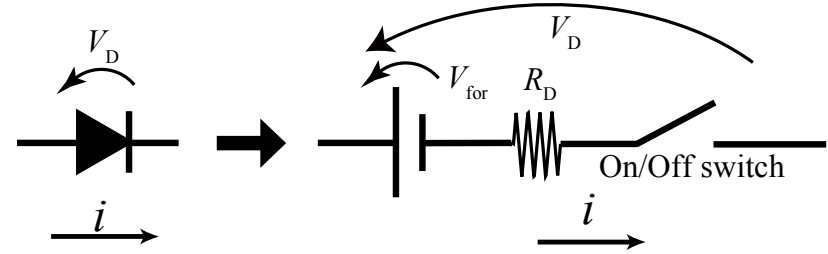

(a) Dynamic model

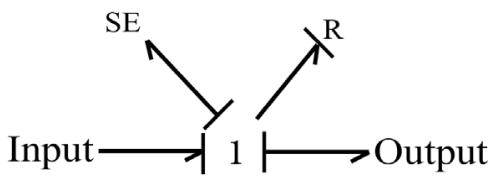

(b) Bond-graph representation

Fig. 5 Diode model.

\subsubsection{Diode-bridge model}

The advanced energy-harvester uses the diode-bridge to rectify the current that is generated by the piezoelectric transducer. The diode-bridge model can be constructed in Fig. 6(a) using the diode model in Fig. 5(a). Arrows in Fig. 6(a) indicate the positive direction of current. By following the bond-graph rules, Fig. 6(a) can be transformed to the bond-graph model in Fig. 6(b). $R_{1}, R_{2}, R_{3}$, and $R_{4}$ in Fig. 6(b) are variable resistors that can describe the internal resistors and the on/off switches. Using this model, we can simulate full wave rectification.

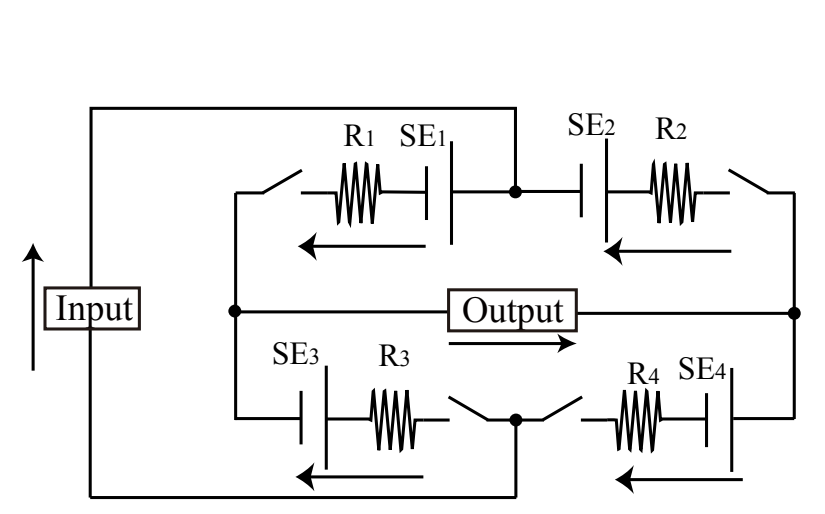

(a) Dynamic model

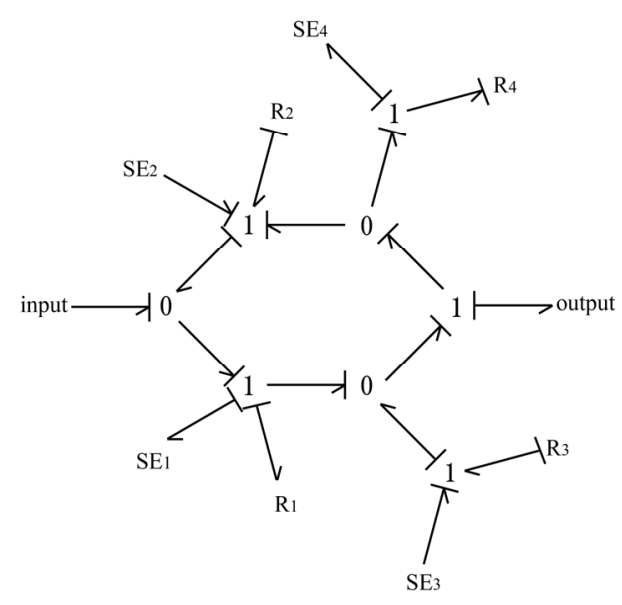

(b) Bond-graph representation

Fig. 6 Diode-bridge model.

\subsection{Bond-graph model of harvesting controller}

The advanced energy-harvester uses the on/off switch to regulate electric current in the circuit. The regulation of current is used for energy-harvesting of the vibrational energy of the vibrating structures. The control operation does not rely on an external control authority but on an internal digital processor. The digital processor is powered by the harvested energy from the advanced harvester. The digital processor controls the on/off switch when the input voltage is more than $2.0 \mathrm{~V}$. Since the voltage generated by the piezoelectric transducer is greater than the input voltage, the advanced energy-harvester uses a DC/DC converter to reduce the input voltage. This converter is driven when input is greater than $5.0 \mathrm{~V}$. When the input voltage is less than $5.0 \mathrm{~V}$, the DC/DC converter does not function. The consumption level of the digital processor has two values. When the DC/DC converter outputs $2.0 \mathrm{~V}$, the digital processor calculates 
the velocity of the mass. The control logic operates such that when the velocity of the mass is 0 , the digital processor closes the on/off switch. The controller model is shown in Fig. 7(a). The energy consumption, both in the DC/DC converter and in the digital processor, is equal to the energy consumption in the equivalent resistance $R_{\mathrm{DC}+\mathrm{MP}}=1.0 \times 10^{3} \Omega$. We refer to an MTF (Modulated TF) element in Fig. 7(a). The MTF element has a variable ratio between inputs and outputs whereas the TF element has a constant proportion. When the input voltage is greater than $5.0 \mathrm{~V}$, the element maintains the output value at $2.0 \mathrm{~V}$. When the input voltage is less than $5.0 \mathrm{~V}$, the element does not provide any output voltage. When the DC/DC converter provides the output power, the digital processor starts control operation, and conducts the state assumption of velocity of the mass. Depending on the velocity, the digital processor outputs a switching signal to the on/off switch in Fig. 7(a). The energy consumption of switching is modelled as the consumption in equivalent resistance $R_{\text {signal }}=1.0 \times 10^{4} \Omega$. The bond-graph model for the circuit in Fig. 7(a) is illustrated in Fig. 7(b).

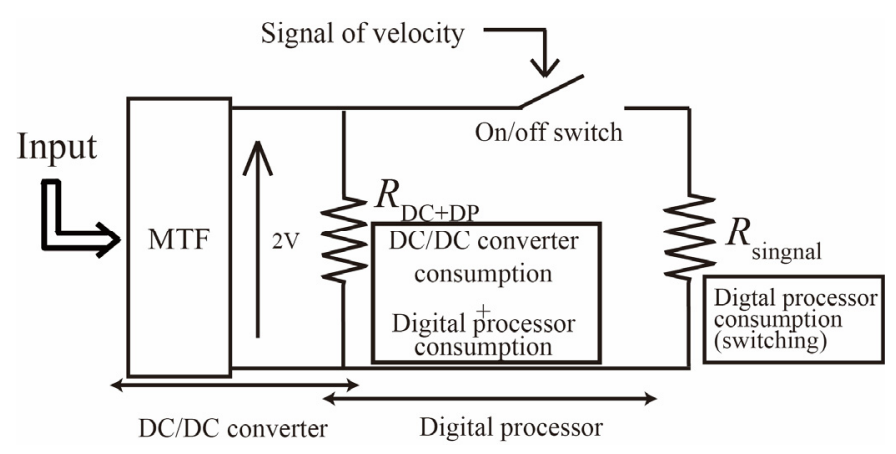

(a) Dynamic model

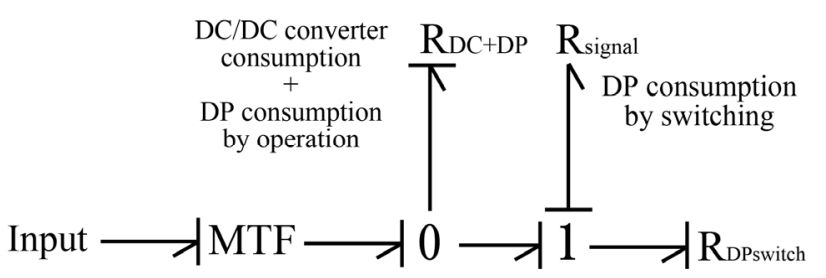

(b) Bond-graph representation

Fig. 7 Harvesting controller model.

\section{Simulation of advanced energy-harvester 5.1. Simulation model}

Figure 8 shows the model of the advanced energy-harvester that has a mass, a piezoelectric transducer, a damper, an on/off switch, a diode-bridge, an energy harvesting circuit, and a harvesting controller. The bond-graph representation of the advanced energy-harvester is illustrated in Fig. 9. Wavy lines represent the feedback control for on/off switching action.

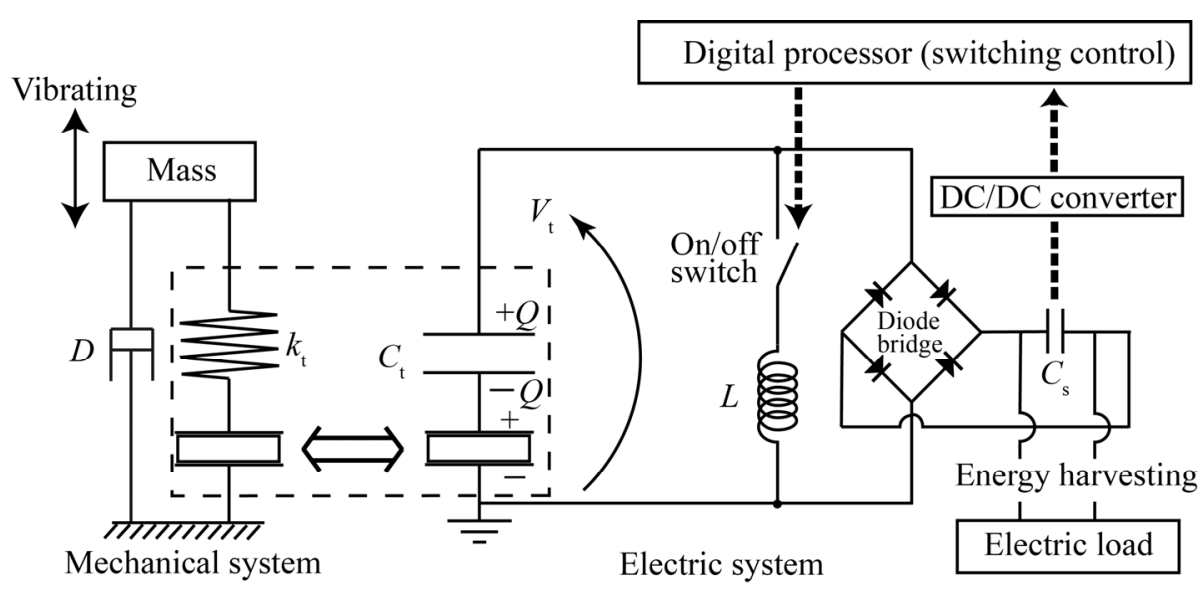

Fig. 8 Advanced energy-harvester model. 


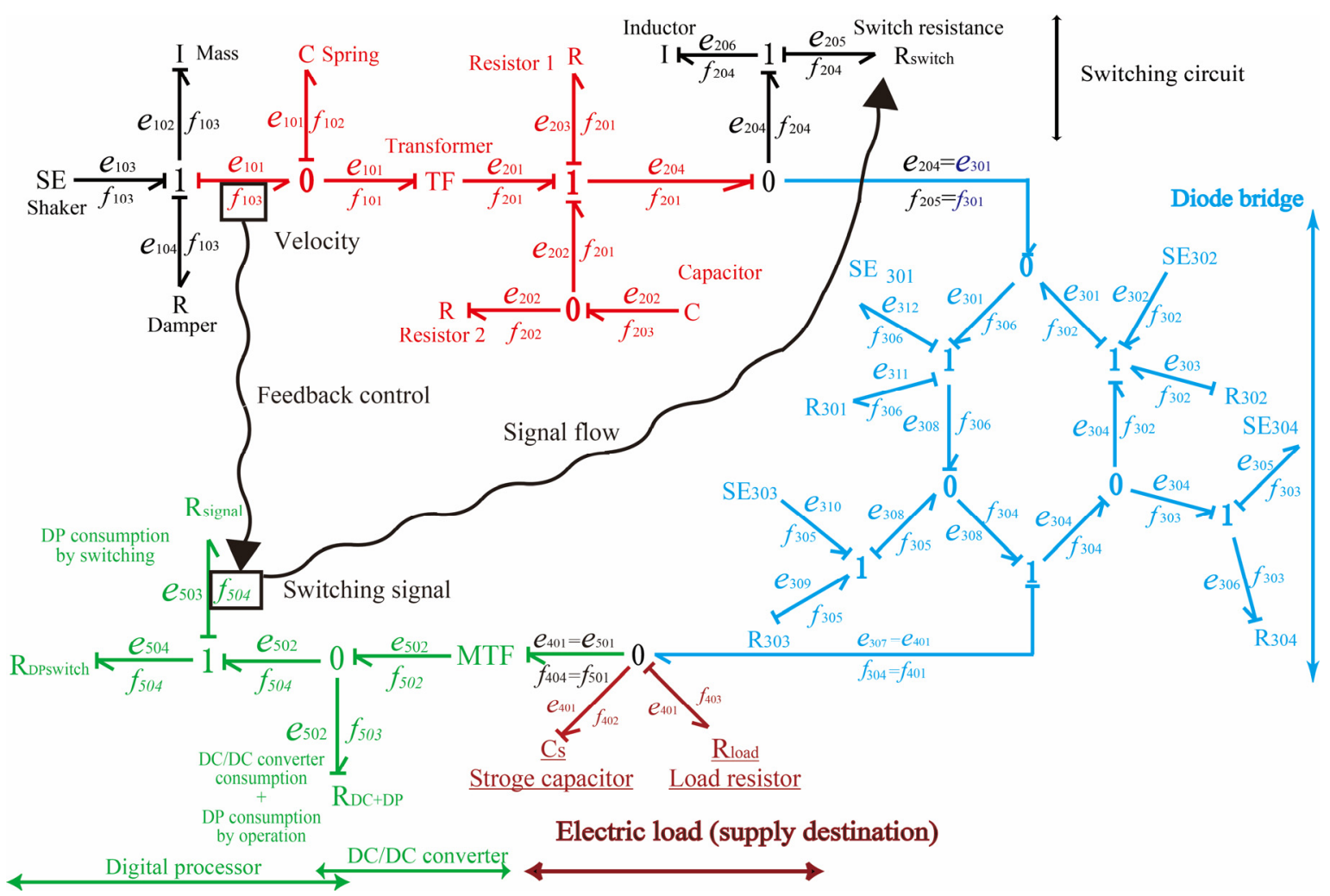

Fig. 9 Whole bond-graph representation of advanced energy-harvester.

\subsection{Simulation results of bond-graph analysis}

Table 4 Parameters of Fig. 8 used in simulation.

\begin{tabular}{c|ccc}
\hline \hline Symbol & Quantity & Value & Unit \\
\hline$C_{\mathrm{S}}$ & Storage capacitance & $4.70 \times 10^{-7}$ & $\mathrm{~F}$ \\
$L$ & Inductance & $2.00 \times 10^{-2}$ & $\mathrm{H}$ \\
$\zeta$ & Damping ratio & $5.40 \times 10^{-3}$ & - \\
$a_{\text {input }}$ & Input acceleration & $3.00 \times 10^{-1}$ & $\mathrm{~m} / \mathrm{s}^{2}$ \\
\hline \hline
\end{tabular}

Some parameters used for the simulations are given in Table 4, where others are listed in Table 3. The on/off switch is a normal off switch. When the velocity of mass is 0 , the on/off switch is closed for $T_{\mathrm{e}} / 2$ seconds, where $T_{\mathrm{e}}$ is natural frequency in the electric circuit and is defined as

$$
T_{\mathrm{e}} \equiv \frac{2 \pi}{\sqrt{L C_{\mathrm{t}}^{\prime}}} .
$$

The basic concept of bond-graph and the way of bond-graph simulation have been described in literature (Karnopp et al. 1990, Thoma 1990), and only a brief explanation is offered here to facilitate understanding of what follows. We obtain simultaneous equations from the bond-graph representation shown in Fig. 9. All efforts and flows are variables. The relations of efforts and flows are determined with the values of bond-graph components, and with current values of variables. When we solve a set of simultaneous equations at time $t$, we obtain all updated values for efforts and flows. Then, when time is incremented to $t+\Delta t$, the set of simultaneous equations is solved again with updated variables.

The performance of the advanced energy-harvester is evaluated by the peak value of harvested power. Thus, we investigate the harvested power for electric loads with different resistances. Power efficiency is the time average of harvested power divided by the time average of input power. Figure 10 shows relationship between power efficiency and different resistance value three seconds after the mass starts to vibrate. The resistance changes from $10^{1}$ to $10^{7} \Omega$. Figure 10 shows that the best power efficiency is $50.4 \%$ when $R_{\mathrm{opt}}=2.37 \times 10^{4} \Omega$. In the small resistance region, the consumption in electric loads is very large. Therefore, the voltage of the storage capacitor cannot rise over $5.0 \mathrm{~V}$. The digital processor is not driven because of the low input. Hence, the advanced energy-harvester cannot function as the 
SSHI system. This makes the power efficiency low. In the large resistance region, because the current almost does not pass through the electric load, the consumption of electric loads is very small. Thus, the power efficiency is also low.

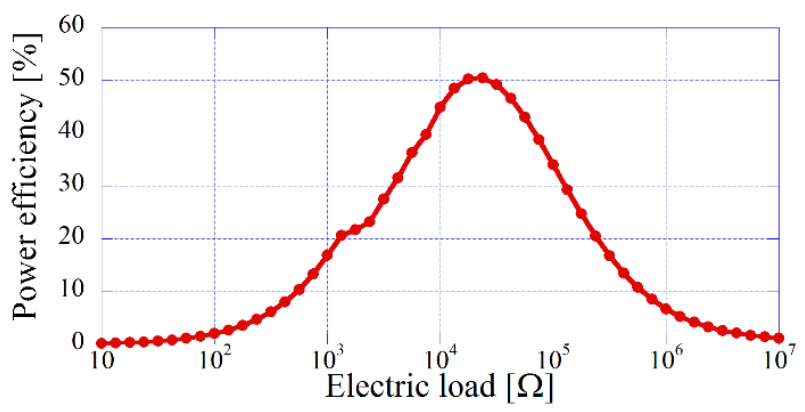

Fig. 10 Power efficiency vs. various resistances.

We consider the dynamics of the most efficient harvester that uses the optimal resistor. Figure 11 shows the voltages of the piezoelectric transducer and the storage capacitor. When the voltage of the storage capacitor is greater than $5.0 \mathrm{~V}$, the voltage waveform of the piezoelectric transducer changes dramatically. The DC/DC converter allows the current to flow into the digital processor; thus, the voltages of the piezoelectric transducer and the storage capacitor increases as a result of electrical vibrations in the LCR circuit by switching operation.

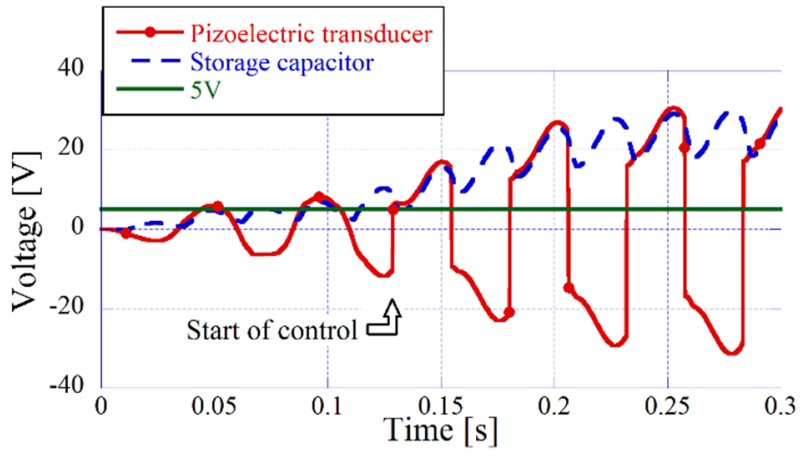

Fig. 11 History of piezoelectric transducer and storage capacitor voltages.

We also consider the power values at steady state: input power $P_{\text {input }}$ by the external force $F_{\text {ext }}$, mechanical power $P_{\text {mech }}$, electric power $P_{\text {elec }}$, diode-bridge power $P_{\text {bridge }}$, electric load circuit power $P_{\text {load }}$, and controller power $P_{\text {control }}$. These values are defined as

$$
\begin{aligned}
& P_{\text {input }} \equiv F_{\text {ext }} \dot{x}, \\
& P_{\text {mech }} \equiv M \dot{x} \ddot{x}+\left(k_{\mathrm{t}}-b_{\mathrm{t}}{ }^{2} C_{\mathrm{t}}^{\prime}\right) x \dot{x}+D \dot{x}^{2}, \\
& P_{\text {elec }} \equiv L i_{204} i_{204}+C_{\mathrm{t}}^{\prime} V_{202} \dot{V}_{202}+R_{\text {switch }} i_{204}{ }^{2}, \\
& P_{\text {bridge }} \equiv\left(-V_{302} i_{302}+V_{305} i_{303}-V_{310} i_{305}+V_{312} i_{306}\right)+\left(R_{301} i_{306}{ }^{2}+R_{302} i_{302}{ }^{2}+R_{303} i_{305}{ }^{2}+R_{304} i_{303}{ }^{2}\right), \\
& P_{\text {load }} \equiv C_{\mathrm{s}} V_{401} \dot{V}_{401}+R_{\text {load }} i_{403}{ }^{2}, \\
& P_{\text {control }} \equiv R_{\mathrm{DC}+\mathrm{DP}} i_{503}{ }^{2}+R_{\text {signal }} i_{504}{ }^{2}+R_{\mathrm{DPswith}} i_{504}{ }^{2},
\end{aligned}
$$

respectively, where $\dot{x}=f_{103}$ and $F_{\text {ext }}=e_{103}$. Mass velocity $\dot{x}$ and current $i$ are flow variables $(e)$, and voltage $V$ is a effort variable (f), in Fig. 9. The subscripts of variables are shown in Fig. 9. Since the input power is divided into five parts, the input power is equal the summation of the five power components. Thus, the relational expression is

$$
P_{\text {input }}=P_{\text {total }}\left(\equiv P_{\text {mech }}+P_{\text {elec }}+P_{\text {bridge }}+P_{\text {load }}+P_{\text {control }}\right) \text {. }
$$

Figure 12 shows the history of significant voltages and powers. When the voltage of the piezoelectric transducer 
is inverted $(t=1.049 \mathrm{~s})$, the mechanical power is not smooth. The electric and the electric load circuit powers have a discontinuity. When the voltage of the storage capacitor changes to the rise state from the drop state $(t=1.053 \mathrm{~s})$, the electric and the electric load circuit powers are interchanged. From $1.049 \mathrm{~s}$ to $1.053 \mathrm{~s}$, the voltage of the storage capacitor is higher than the voltage of the piezoelectric transducer; therefore, current does not pass through the electric load. When the voltage of the piezoelectric transducer is equal to the sum of the storage-capacitor voltage and the diode-bridge forward voltage, current flows through the electric-load. Thus, the electric load circuit power increases and the electric power decreases. Since Eqs. (16) and (18) have derivatives of electrical values, such as $i$ and $\dot{V}$, these power lines are discontinuous.

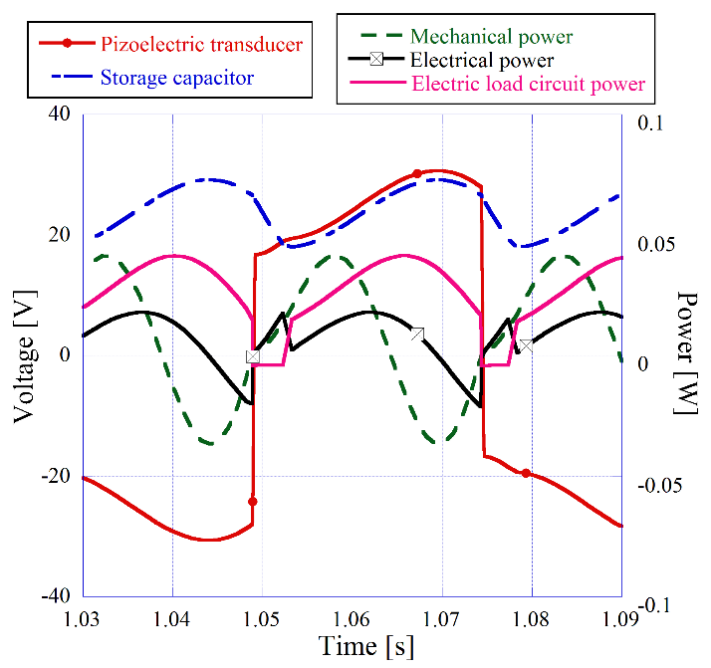

Fig. 12 History of various powers at steady state.

Figure 13 shows the input and the total power values. The total power includes the dissipated power. We can confirm that the total power equals the input power in Fig. 13, which leads to the validity of the whole bond-graph model of Fig. 9. Based on the results we presented so far, we can see that the simulation results of the advanced energy-harvester support our claims.

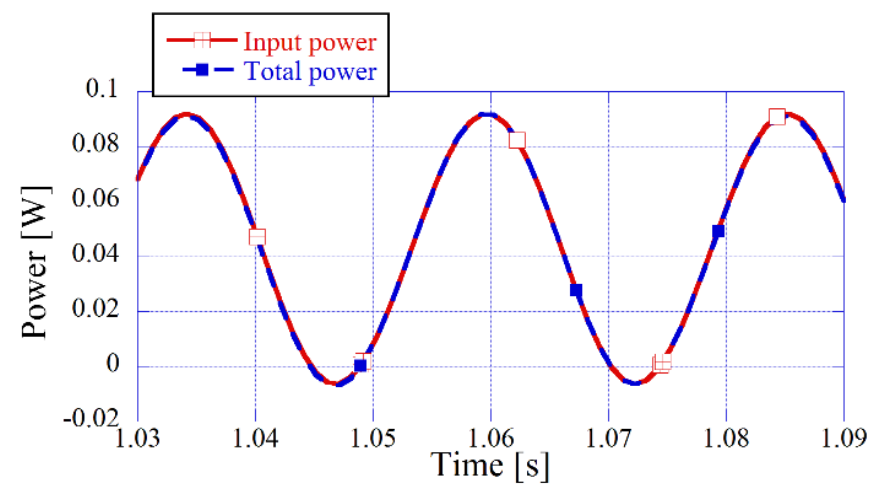

Fig. 13 History of input and total power at steady state.

\section{Conclusions}

We evaluated the performance of the advanced energy-harvester using the bond-graph method, which is a graphical analysis technique. The harvester is controlled by a digital processor which is in turn powered by the harvested energy. To create the bond-graph model of the advanced energy-harvester, we first modeled the piezoelectric transducer, the diode-bridge, and the controller. Next, we investigated the validity and the utility of the piezoelectric transducer model. Numerical simulations showed that the harvested power efficiency was strongly influenced by the resistance of the electric load. For small and large resistances, the power efficiency was low because the voltage of the storage capacitor could not rise owing to the large consumption at the electric load or small provision to electronics. We also investigated the dynamics of the advanced energy-harvester. When the digital processor started to function, the 
voltage waveform of the piezoelectric transducer greatly changed from the original one and was affected by the switching operation. Furthermore, we studied the evolution of different types of powers in the advanced energy-harvester. Because the electric charge was discontinuous, some power waveforms also became discontinuous. Finally, bond-graph simulations demonstrated that the input power was composed of different powers and that the total power equaled the input power. We hope that the bond-graph method will be utilized extensively for energy-harvesting analyses.

\section{Acknowledgements}

This research was supported by Grant-in-Aid for Young Scientists (A) (No. 23686125) and Challenging Exploratory Research (No. 25630436) from the Japan Society for the Promotion of Science. This work was partly supported by the JSPS Core-to-Core Program, A. Advanced Research Networks, "International research core on smart layered materials and structures for energy saving".

\section{References}

Adachi, K. and Sakamoto, T., Study on energy transfer efficiency analysis of cantilever type of piezocomposite vibration energy harvester, Transactions of the Japan Society of Mechanical Engineers Series C, Vol. 78, No. 789 (2012), pp. 1575-1585 (in Japanese).

Badel, A., Guyomar, D., Lefeuvre, E. and Richard, C., Efficiency enhancement of a piezoelectric energy harvesting device in pulsed operation by synchronous charge inversion, Journal of Intelligent Material Systems and Structures, Vol. 16, No. 10 (2005), pp. 889-901.

Busch-Vishniac, I. J. and Paynter, H. M., Bond graph models of acoustical transducers, Journal of the Franklin Institute, Vol. 328, Nos. 5-6 (1991), pp. 663-673.

Caliò, R., Rongala, U. B., Camboni, D., Milazzo, M., Stefanini, C., de Petris, G. and Oddo, C. M., Piezoelectric energy harvesting solutions, Sensors, Vol. 14, No. 3 (2014), pp. 4755-4790.

Dicken, J., Mitcheson, P. D., Stoianov, I. and Yeatman, E. M., Power-extraction circuits for piezoelectric energy harvesters in miniature and low-power applications, IEEE Transactions on Power Electronics, Vol. 27, No. 11 (2012), pp. 4514-4529.

Jaeger, R. and Blalock, T., Microelectronic Circuit Design $4^{\text {th }}$ ed. (2010), McGraw-Hill, New York.

Karnopp, D. C., Margolis, D. L. and Rosenberg, R. C., System Dynamics: A Unified Approach 2 ${ }^{\text {nd }}$ ed. (1990), John Wiley \& Sons, Inc., New York.

Makihara, K., Miyakawa, T. and Onoda, J., Low energy dissipation electric circuit for energy harvester, Smart Materials and Structures, Vol. 15, No. 5 (2006), pp. 1493-1498.

Makihara, K., Takeuchi, S., Shimose, S., and Onoda, J., Portable power scavenging from structural vibrations using autonomous self-powered device, Transactions of JSASS, Space Technology Japan, Vol. 10, No. 28, (2012a) pp. $13-18$.

Makihara, K., Onoda, J., Shimose, S. and Takeuchi, S., Innovative digital self-powered autonomous system for multimodal vibration suppression, AIAA Journal, Vol. 50, No. 9 (2012b), pp. 2004-2011.

Mason, W. P., Electromechanical Transducers and Wave Filters $2^{\text {nd }}$ ed. (1948), Van Nostrand, New York.

Masuda, A., Senda, A., Sanada, T. and Sone, A., Global stabilization of high-energy response for a duffing-type wideband nonlinear energy harvester via self-excitation and entrainment, Journal of Intelligent Material Systems and Structures, Vol. 24, No. 13 (2013), pp. 1598-1612.

Niederberger, D. and Morari, M., An autonomous shunt circuit for vibration damping, Smart Materials and Structures, Vol. 15, No. 2, (2006) pp. 359-364.

Rodriguez-Fortun, J. M., Orus, J., Buil, F. and Castellanos, J. A., General bond graph model for piezoelectric actuators and methodology for experimental identification, Mechatronics, Vol. 20, No. 2 (2010), pp. 303-314.

Thoma, J. U., Simulatoin by Bondgraphs (1990), Springer-Verlag, Berlin Heidelberg.

Wang, W., Shin, D., Han, C. and Choi, H., Modeling and simulation for dual stage system using bond graph theory, International Symposium on Optomechatronic Technologies (2009), pp. 197-202.

Yamamoto, Y. and Makihara, K., Development of wireless health monitoring system for isolated space structures, Transactions of JSASS, Space Technology Japan, Vol. 12 (2014), pp. 55-60. 